\title{
Combined analysis of HPV DNA, p16, p21 and p53 to predict prognosis in patients with stage IV hypopharyngeal carcinoma
}

\author{
Perle Ernoux-Neufcoeur $\cdot$ Mohammad Arafa $\cdot$ Christine Decaestecker $\cdot$ \\ Anaëlle Duray $\cdot$ Myriam Remmelink $\cdot$ Xavier Leroy $\cdot$ Michael Herfs \\ Joan Somja $\cdot$ Christophe E. Depuydt $\cdot$ Philippe Delvenne $\cdot$ Sven Saussez
}

Received: 26 February 2010 / Accepted: 19 March 2010 / Published online: 8 April 2010

(c) The Author(s) 2010. This article is published with open access at Springerlink.com

\begin{abstract}
Purpose We examined p16, p21 and p53 expression in combination with the presence of human papillomavirus (HPV) DNA as molecular markers to predict survival in patients with stage IV hypopharyngeal squamous cell carcinoma (HSCC).

Methods Paraffin-embedded tumours from HSCC patients $(n=75)$ were evaluated for p16, p21 and p53 expression by immunohistochemistry. HPV DNA was detected by GP5+/6+ consensus PCR and subsequent genotyping by E6/E7 type-specific PCR for HPV types 6, 11, $16,18,31,33,35,39,45,51,52,53,56,58,59,66$ and 68.
\end{abstract}

P. Ernoux-Neufcoeur and M. Arfa have contributed equally to this work.

C. Decaestecker and P. Delvenne are Senior Research Associates with the Belgian National Fund for Scientific Research (FNRS, Brussels, Belgium).

P. Ernoux-Neufcoeur and A. Duray are Ph.D. students supported by a grant from the FNRS (Bourse Télévie).

P. Ernoux-Neufcoeur · A. Duray $\cdot$ S. Saussez $(\varangle)$

Laboratory of Anatomy, Faculty of Medicine and Pharmacy,

University of Mons, Pentagone 2A-Avenue du Champ de Mars, 6,

B-7000 Mons, Belgium

e-mail: sven.saussez@umons.ac.be

M. Arafa $\cdot$ M. Herfs · J. Somja $\cdot$ P. Delvenne

Department of Pathology, CHU Sart-Tilman,

University of Liège, Liège, Belgium

C. Decaestecker

Laboratory of Toxicology, Institute of Pharmacy,

Université Libre de Bruxelles, Brussels, Belgium

M. Remmelink

Department of Pathology, Hôpital Erasme,

Université Libre de Bruxelles, Brussels, Belgium
Results Among the 61 specimens that tested positive for the $\beta$-globin, HPV typing identified 50 patients with highrisk (hr) HPV types. HPV 16E7 DNA was detected in 74\% (37 cases) of these specimens. Twelve patients were found to be infected with multiple HPV types. However, the presence of hrHPV DNA was not found to correlate with the proportion of disease-free patients. The 5-year disease-free survival rate was $73 \%$ in p53- tumours versus $48 \%$ in p53+ tumours $(P=0.008)$.

Conclusion In our series of patients with stage IV HSCC, the hrHPV+ subgroup had a similar prognosis (in terms of recurrence risk) as the HPV- subgroup. p53 overexpression was associated with a worse prognosis.

Keywords Human papillomavirus · PCR · Typing · Hypopharynx $\cdot$ Carcinoma

\section{Introduction}

Head and neck squamous cell carcinoma (HNSCC) represents the sixth most common form of cancer that is

\author{
X. Leroy \\ Department of Pathology, Faculty of Medicine, \\ Hôpital Claude Huriez and Centre de Biologie-Pathologie-CHRU, \\ Lille, France \\ C. E. Depuydt \\ Laboratory for Clinical Pathology and Molecular Biology \\ (Labo Lokeren, campus Riatol), Antwerp, Belgium \\ S. Saussez \\ Department of Oto-Rhino-Laryngology, CHU Saint-Pierre, \\ Université Libre de Bruxelles, Brussels, Belgium
}


diagnosed worldwide. It is the most common malignant neoplasm that arises from the mucosa of the upper aerodigestive tract (Grandis et al. 2004; Hunter et al. 2005). During the past 30 years, survival rates have not improved significantly for this type of cancer, and $50 \%$ of HNSCC patients still eventually die of their disease and 5\% develop additional primary tumours each year (Grandis et al. 2004; Hunter et al. 2005). Furthermore, HNSCC has a large impact on the quality of life of all HNSCC patients and survivors (Bernier et al. 2004; Cooper et al. 2004). Significant morbidity caused by the treatments often mandates longterm multidisciplinary care, which leads to increased financial burdens for institutions that treat HNSCC patients (Hunter et al. 2005). The management of head and neck cancer has become increasingly complex in recent years due to combined-modality treatment regimens as well as the integration of new diagnostic and therapeutic technologies. Nearly two-thirds of HNSCC patients present with advanced disease (stages III and IV). Despite the use of resection and concomitant post-operative chemotherapy and radiotherapy, these advanced HNSCCs frequently recur in the original tumour beds (Hunter et al. 2005; Schantz et al. 1997). Patients with stage IV hypopharyngeal carcinoma represent one of the HNSCC subgroups with the worst prognosis, as the 5-year survival rate for this population is less than $15 \%$.

Biological markers are therefore required to identify the high-risk HNSCC patients who are in need of highly aggressive treatments after surgical resection of their tumours (Grandis et al. 2004). Over the last 10 years, it has become clear that there is a subset of oropharyngeal squamous cell carcinomas (OSCCs) that is associated with highrisk human papillomavirus (hrHPV) infection, in particular HPV type 16 (D'Souza et al. 2007). HPV-positive OSCCs seem to be different from HPV-negative tumours with respect to tumour differentiation, risk factors and the genetic changes that are present, indicating that HPVpositive OSCCs represent a separate tumour entity from HPV-negative OSCCs, which have specific molecular pathways associated with their tumourigenicity (Reimers et al. 2007). A recent case-control study comparing 100 patients with OSCC and 200 control patients demonstrated that oral HPV infection was strongly associated with oropharyngeal carcinoma among patients who did not have the classical risk factors of tobacco and alcohol use (D'Souza et al. 2007). This study also demonstrated that a high lifetime number of oral sex or vaginal sex partners, engagement in casual sex, early age at first intercourse and infrequent use of condoms were all associated with HPV-16-positive oropharyngeal cancer (D'Souza et al. 2007).

Molecular studies of cervical carcinoma, the cancer that has the most widely accepted association with HPV, have shown that after integration of the virus, the expression of the transcription factor E2 (which represses the transcription of E6 and E7 oncogenes) is disrupted. As a consequence of this disruption, the E6 and E7 genes are transcribe and result in the production of oncoproteins that bind to and degrade the p53 and retinoblastoma $(\mathrm{Rb})$ tumour suppressor, respectively. The p16 protein functions as a tumour suppressor by binding to the cyclin D1 CDK4/CDK6 complex, thereby preventing phosphorylation of the $\mathrm{Rb}$ protein. $\mathrm{Rb}$ downregulation subsequently results in p16 upregulation (Weinberger et al. 2006). Begum et al. (2003) have shown that p16 overexpression is a marker of oropharyngeal origin and has association with HPV infection in HNSCC tumours. In contrast, in tobacco-related HNSCCs, the loss of the tumour-suppressor p16 protein is a common and early event in tumourigenesis (Reed et al. 1996). Several studies that were dedicated to studying oral and oropharyngeal carcinomas have demonstrated that HPV-positive patients have a better prognosis than HPV-negative patients (Ritchie et al. 2003; Weinberger et al. 2006). A more recent study showed that HPV-16 copy number was positively associated with response to concomitant chemoradiotherapy and with better overall and disease-specific survival (Kumar et al. 2007). Moreover, joint assessment of p53 expression and HPV infection seems to provide an excellent indicator of prognosis (Smith et al. 2008). In a large series of 294 HNSCCs with 10 years of follow-up, Smith et al. (2008) observed that patients with tumours that were p53-/ hrHPV+ had the highest survival and lowest recurrence rates, whereas patients with tumours that were p53+l HPV - had significantly worse outcomes.

Despite their promise as prognostic markers for patients with OSCC, no studies have been performed to analyse the prognostic implication of this combination of markers in patients with hypopharyngeal carcinoma. In the current study, we examined the clinical utility of the combination of the most promising predictive markers for OSCC, including HPV DNA detection as well as p16, p21 and p53 expression in a homogeneous series of 75 patients with stage IV hypopharyngeal SCC.

\section{Materials and methods}

Histopathological and clinical data

Formalin-fixed, paraffin-embedded hypopharyngeal squamous cell carcinoma (HSCC) tumour specimens were obtained from 4 female and 71 male patients who underwent radical curative-intent surgery between January 1996 and December 2000 in the E.N.T. Department of the Hôpital Claude Huriez (Lille, France). For each surgical specimens, we have selected the paraffin block presenting the 
higher proportion of carcinoma (avoiding necrotic area frequently observed in the central part of the tumour). Clinical staging was performed according to the TNM classification system (Wittekind et al. 2004), and data regarding the tumours of the 75 stage IV hypopharyngeal patients included in this study are detailed in Table 1 . The diagnoses were established based on the histological criteria previously described by Hyams et al. (1988). All hypopharyngeal SCCs included in this study were primary tumours, and the patients did not have distant metastases or recurrences. The tumours were categorised as well-differentiated $(n=38)$, moderately differentiated $(n=26)$ and poorly differentiated $(n=11)$. This retrospective study was approved by the local Institutional Review Board.

All of the hypopharyngeal SCC specimens used in this study came from patients who did not undergo chemotherapy and/or radiotherapy before surgery. After surgery, all patients were treated with standard post-operative radiotherapy, but not chemotherapy. All patients used alcohol and tobacco. Thus, the 75 stage IV hypopharyngeal SCC specimens that were included in this study comprised a very homogeneous sample, both clinically and histopathologically.

\section{DNA extraction}

The formalin-fixed, paraffin-embedded tissue samples were sectioned $(10 \times 5 \mu \mathrm{m})$, deparaffinised and digested with proteinase $\mathrm{K}$ by overnight incubation at $56^{\circ} \mathrm{C}$. DNA was purified using the QIAamp DNA Mini Kit (Qiagen, Benelux, Belgium) according to the protocol recommended by the manufacturer.

Detection of HPV by polymerase chain reaction (PCR) amplification

HPV detection was performed by PCR using GP5+/GP6+ primers (synthesised by Eurogentec, Liege, Belgium). These GP5+/GP6+ primers amplify a consensus region located within L1 region of the HPV genome. PCR for HPV-L1 DNA amplification was performed in $25 \mu \mathrm{l}$ of a reaction mixture containing $2 \mu \mathrm{l}$ of extracted DNA, $2.5 \mu \mathrm{l}$ $1 \times$ PCR buffer, $0.025 \mathrm{U}$ Taq DNA polymerase (Roche, Mannheim, Germany), $200 \mu \mathrm{M}$ dNTPs and $0.5 \mathrm{pmol}$ of each primer. The cycling conditions for PCR were as follows: denaturation was performed at $94^{\circ} \mathrm{C}$ for $1 \mathrm{~min}$, annealing was performed at $40^{\circ} \mathrm{C}$ for $1 \mathrm{~min} 30 \mathrm{~s}$ and extension was performed at $72^{\circ} \mathrm{C}$ for 2 min for a total of 40 amplification cycles. The first cycle was preceded by a 3 -min denaturation step at $94^{\circ} \mathrm{C}$. The last cycle was followed by an additional 5 -min extension step at $72^{\circ} \mathrm{C}$. Aliquots $(10 \mu \mathrm{l})$ of each PCR product were electrophoresed through a $1.8 \%$ agarose gel and stained with ethidium bromide to
Table 1 Patient characteristics

Mean age: 55 years (range $40-78$ )

Gender

Male: 71 patients $(95 \%)$

Female: 4 patients $(5 \%)$

Localisation

Piriform sinus: 56 cases $(75 \%)$

Postcricoid area: 16 cases $(21 \%)$

Posterior wall: 3 cases (4\%)

Grade

Well differentiated: 38 cases (51\%)

Moderately differentiated: 26 cases (34\%)

Poorly differentiated: 11 cases $(15 \%)$

TNM stage: ( $n=75$ cases of stage IV disease)

T2N2: 8 cases

T3N2: 7 cases

T4N0: 12 cases

T4N1: 6 cases

T4N2: 39 cases

T4N3: 3 cases

Treatment

Partial pharyngolaryngectomy: 9 cases

Total pharyngolaryngectomy: 49 cases

Circular pharyngolaryngectomy: 8 cases

Esopharyngolaryngectomy: 9 cases

28 presented larynx cartilage invasion

112 neck dissections in 75 patients

47 patients with extranodal spread

All patients were treated with standard post-operative

radiotherapy, but did not receive post-operative chemotherapy.

Recurrence: $(n=23)$

Local recurrence: 17 cases

Distant recurrence: 11 cases

Follow-up: (72 patients had clinical follow-up data available)

9 second primary cancers: 6 cases of lung cancer,

1 case of prostate cancer, 1 case of kidney cancer

6 head and neck second primaries

29 deaths, including 20 caused by the HNSCC and 9 deaths that were unrelated to HNSCC ( 5 caused by a second primary cancer, 3 caused by medical disease, and 1 from unknown causes)

allow for visualisation of the amplified HPV-L1 DNA fragments.

Real-time quantitative PCR amplification of type-specific HPV DNA

All DNA extracts were tested for the presence of 18 different HPV genotypes using TaqMan-based real-time quantitative PCR targeting type-specific sequences of the following viral genes: $6 \mathrm{E} 6,11 \mathrm{E} 6,16 \mathrm{E} 7,18 \mathrm{E} 7,31 \mathrm{E} 6,33$ 
E6, 35 E6, 39 E7, 45 E7, 51 E6, 52 E7, 53 E6, 56 E7, 58 E6, 59 E7, 66 E6, $67 \mathrm{~L} 1$ and 68 E7 (Depuydt et al. 2006). For the different real-time quantitative PCR assays, the analytical sensitivity ranged from 1 to 100 copies and was calculated using standard curves for 18 type-specific PCRs constructed with plasmids containing the entire genome of the different HPV types (Depuydt et al. 2007). Real-time quantitative PCR for the detection of $\beta$-globin was performed in each PCR assay to verify the quality of the DNA in the samples and to measure the amount of input DNA (Arbyn et al. 2009; Depuydt et al. 2007).

The following HPV types that were tested were considered to be high-risk (hr): 16, 18, 31, 33, 35, 39, 45, 51, 52, 53, 56, 58, 59 and 66.

\section{Immunohistochemistry}

All tumour samples were fixed in $10 \%$ buffered formaldehyde for $24 \mathrm{~h}$, dehydrated and embedded in paraffin. Immunohistochemistry was performed on 5 - $\mu$ m-thick sections mounted on silane-coated glass slides (Saussez et al. 2008). Before starting the immunohistochemistry protocol, deparaffinised tissue sections were placed in a $0.01 \mathrm{M}$ citrate buffer (pH 6.0) and briefly pre-treated in a microwave for $2 \times 5 \mathrm{~min}$ at $900 \mathrm{~W}$. The sections were then incubated with a solution of $0.4 \%$ hydrogen peroxide for $5 \mathrm{~min}$ to block endogenous peroxidase activity, rinsed in phosphatebuffered saline (PBS; $0.04 \mathrm{M} \mathrm{Na}_{2} \mathrm{HPO}_{4}, 0.01 \mathrm{M} \mathrm{KH}_{2} \mathrm{PO}_{4}$ and $0.12 \mathrm{M} \mathrm{NaCl}, \mathrm{pH} 7.4$ ) and successively exposed to solutions containing avidin $(0.1 \mathrm{mg} / \mathrm{ml}$ in PBS) and biotin $(0.1 \mathrm{mg} / \mathrm{ml}$ in PBS) for 20 -min periods to avoid falsepositive staining reactions resulting from the presence of endogenous biotin. After a thorough washing with PBS, the sections were incubated for $20 \mathrm{~min}$ with a solution of $0.5 \%$ casein in PBS and sequentially exposed to the following solutions: (1) the specific primary antibody; (2) the corresponding biotinylated secondary antibody (polyclonal goat anti-rabbit IgG); (3) the avidin-biotin-peroxidase complex (ABC kit), all at room temperature. The samples were subjected to thorough washing steps to remove unbound proteins in between incubation steps. The antigen-dependent presence of the peroxidase complex in the sections was visualised by incubation with the chromogenic substrates containing diaminobenzidine and $\mathrm{H}_{2} \mathrm{O}_{2}$. After rinsing, the sections were counterstained with luxol fast blue and mounted in a synthetic medium. To exclude antigenindependent staining, the incubation step with primary/secondary antibodies was omitted from the protocol in control samples. In all instances, these controls were negative. The biotinylated secondary antibodies and $\mathrm{ABC}$ kit were obtained from DakoCytomation (Glostrup, Denmark). The p16, p21 and p53 antibodies came, respectively, from Abcam (for p16 and p21, Cambridge, UK) and DakoCytomation
(Glostrup, Denmark). Assessment of p16, p21 and p53 immunoreactivities was performed by two investigators who were blinded to the clinical details of the patients. Tumours were classified in a binary manner as either p16-, p21- and p53-positive (strong, diffuse staining) or p16-, p21- and p53-negative (weak or absent staining; i.e., the labelling index (LI) corresponding to the percentage of immunopositive cells was $\leq 5 \%$ ).

Data analysis

Categorical data from independent groups were compared using the chi-square test or Fisher's exact test as appropriate. Survival was measured in months from the date of diagnosis until death or until the date at which patient was last known to be alive. The standard survival time analyses were performed using Kaplan-Meier curves and the Gehan generalised Wilcoxon and log-rank tests. The statistical analyses were performed using the Statistica software package (Statsoft, Tulsa, USA).

\section{Results}

HPV status as determined by the GP5+/GP6+ consensus primer and by real-time quantitative PCR amplification of type-specific HPV DNA and correlation with clinicopathological parameters

A total of eight cases of the 75 specimens were found to have insufficient tissue quantities available for DNA extraction or quantitative PCR after pathological evaluation and immunohistochemistry and were therefore excluded from further analysis (Fig. 1). Out of the remaining 67 cases, another six cases, which were $\beta$-globin PCR-negative, were also excluded from further analysis. Ultimately, $61 \beta$-globin PCR-positive specimens were typed using quantitative real-time PCR using primers for 18 different HPV types (Fig. 1). From this homogenous group of 61 HSCC tumour specimens, we identified 50 patients $(82 \%)$ whose tumours tested positive for the following hrHPV types: HPV 16 (37 cases), 18 ( 4 cases), 33 (11 cases), 39 ( 1 case), 51 (5 cases), 53 ( 1 case), 58 ( 2 cases), 59 ( 1 case) and 66 (4 cases). Twelve patients were infected with multiple types of hrHPV. In the hrHPV - negative subgroup $(n=11)$, four patients tested positive for HPV infection using the GP5+/ GP6+ consensus primers and were considered to be infected with low-risk (lr) HPV types (Fig. 1). Only seven tumours were negative for both GP5+/GP6+ and typespecific HPV PCR analysis (11\%). Among the 50 patients with hrHPV+ tumours, 38 tumours were both GP5+/GP6+positive and type-specific HPV-positive (hrHPV+ group). However, 12 tumours were GP5+/GP6+-negative and 
Fig. 1 Flow diagram of the processing and HPV PCR results from formalin-fixed, paraffinembedded hypopharyngeal carcinoma specimens included in this study. Archived tissue blocks were obtained from 75 patients who underwent curative-intent surgery for stage IV hypopharyngeal carcinoma between January 1996 and December 2000. Eight samples could not be analysed due to insufficient material, and in another six samples, $\beta$-globin could not be amplified. Therefore, 61 cases were analysed by type-specific real-time PCR. Among these patients, $82 \%$ tested positive for infection with one or several types of hrHPVs, $7 \%$ were positive for lrHPV and $11 \%$ were HPV -. Among the 50 patients with hrHPV+ tumours, 38 tumours were both GP5+/ GP6+-positive and type-specific HPV-positive (hrHPV+ group). However, 12 tumours were GP5+/GP6+-negative and type-specific HPV-positive, corresponding to an integrated $\mathrm{HPV}+$ group (int. hrHPV+)

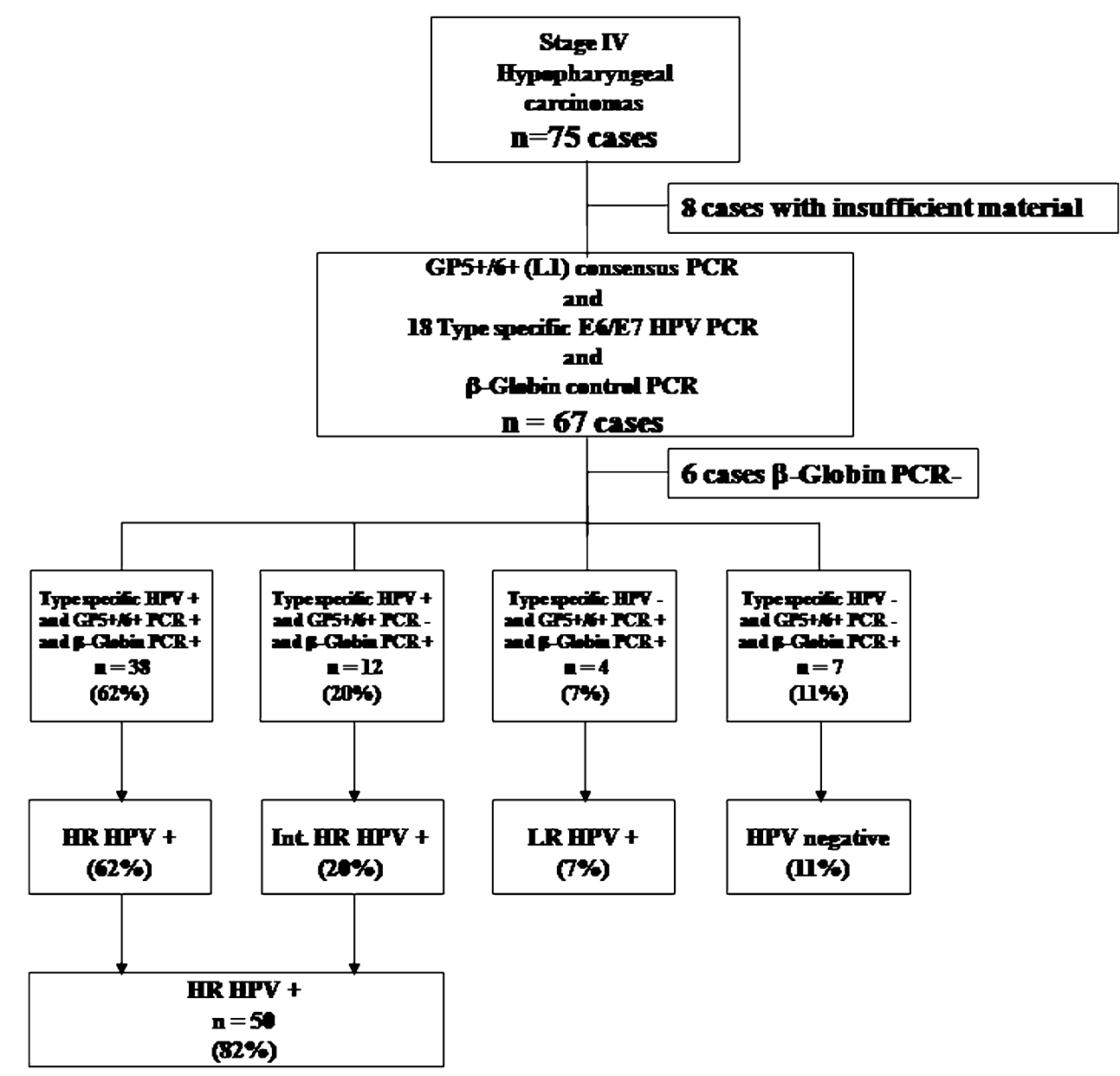

type-specific HPV-positive, corresponding to an integrated $\mathrm{HPV}+$ group (int. hrHPV+) (Fig. 1).

The mean age and the sex ratio of the patients with hrHPV+ tumours (50 cases) were both identical to those of the patients with HPV- or lrHPV+ tumours (11 cases). Statistical analysis did not reveal a significant correlation between hrHPV positivity (as determined by E6/E7 real-time quantitative PCR amplification), and the percentage of tumours that were poorly differentiated or had lymph node involvement, capsular effraction or laryngeal cartilage invasion (Table 2). The hrHPV positivity was not found to be correlated with the proportion of disease-free patients in our series of patients with stage IV hypopharyngeal carcinoma (Fig. 2). However, 32\% (16/50 cases) of patients with hrHPV+ tumours recurred, when compared to $8 \%$ of patients with HPV - or lrHPV+ tumours. The 5-year disease-free survival was $88 \%$ in HPV- or lrHPV+ tumours versus $58 \%$ in hrHPV+ tumours (log-rank test, NS).

p16 expression as determined by immunohistochemistry (IHC)

Immunohistochemical expression of p16 was detected in 7 (9\%) out of 75 tumours (Fig. 3a,b,c). As visualised in
Fig. 3b, p16 displayed a diffuse cytoplasmic/nuclear pattern of expression that was dichotomous [highly expressed (Fig. 3b) versus low/no expression (Fig. 3a)]. When all tumours that expressed p16 (7 cases) were compared to those that did not (68 cases), p16 positivity was found to correlate with a better prognosis. In fact, all patients whose tumours expressed p16 were free of disease (Fig. 3c). The 5-year disease-free survival rate was $100 \%$ in p16+ tumours versus $58 \%$ in p16- tumours $(P=\mathrm{NS})$. This difference was not statistically significant, probably due to the small size of the p16+ group (log-rank test: NS). All hrHPV+ patients' tumours expressed p16 (Table 2).

\section{p21 expression as determined by IHC}

Immunohistochemical expression of p21 was detected in $84 \%$ of HSCC specimens (Fig. 4). Moderate-to-intense brown nuclear staining was considered to be a positive result (Fig. 4). The 5-year disease-free survival rate was $60 \%$ in p21+ tumours versus $70 \%$ in p $21-$ tumours. The proportion of disease-free patients was not significantly different between the p21+ and p21- groups (Fig. 4c). Similar rates of p21 positivity were observed in the 
Table 2 Correlation between HPV status and the percentage of patients with lymph node involvement, capsular effraction or cartilage infiltration as well as p16, p21 and p53 expression

\begin{tabular}{|c|c|c|}
\hline$p: N S$ & $\begin{array}{l}\text { Lymph node } \\
\quad \text { involvement: NO }\end{array}$ & $\begin{array}{l}\text { Lymph node } \\
\quad \text { involvement: } N+\end{array}$ \\
\hline hrHPV+ & $11 / 50(22 \%)$ & $39 / 50(78 \%)$ \\
\hline $\mathrm{HPV}-$ or lrHPV+ & $1 / 11(9 \%)$ & $10 / 11(91 \%)$ \\
\hline$p: N S$ & $\begin{array}{l}\text { Capsular effraction: } \\
\quad \text { No }\end{array}$ & $\begin{array}{l}\text { Capsular effraction: } \\
\text { yes }\end{array}$ \\
\hline hrHPV+ & $18 / 48(37.5 \%)$ & $30 / 48(62.5 \%)$ \\
\hline $\mathrm{HPV}-$ or lrHPV+ & $4 / 11(36 \%)$ & $7 / 11(64 \%)$ \\
\hline$p: N S$ & $\begin{array}{l}\text { Cartilage invasion: } \\
\quad \text { No }\end{array}$ & $\begin{array}{l}\text { Cartilage invasion: } \\
\text { yes }\end{array}$ \\
\hline hrHPV+ & $32 / 49(65 \%)$ & $17 / 49(35 \%)$ \\
\hline HPV - or lrHPV+ & $4 / 11(36 \%)$ & $7 / 11(64 \%)$ \\
\hline$p: N S$ & p 16+ & p 16- \\
\hline hrHPV+ & $5 / 46(11 \%)$ & $41 / 46(89 \%)$ \\
\hline HPV - or lrHPV+ & $0 / 10(0 \%)$ & $10 / 10(100 \%)$ \\
\hline$p: N S$ & p $21+$ & p 21- \\
\hline hrHPV+ & $35 / 44(79.5 \%)$ & $9 / 44(20.5 \%)$ \\
\hline $\mathrm{HPV}-$ or lrHPV+ & $9 / 11(82 \%)$ & $2 / 11(18 \%)$ \\
\hline$p: N S$ & p 53+ & p 53- \\
\hline hrHPV+ & $16 / 48(33 \%)$ & $32 / 48(67 \%)$ \\
\hline $\mathrm{HPV}-$ or $1 \mathrm{rHPV}+$ & $4 / 11(36 \%)$ & $7 / 11(64 \%)$ \\
\hline
\end{tabular}

$N S$ not significant

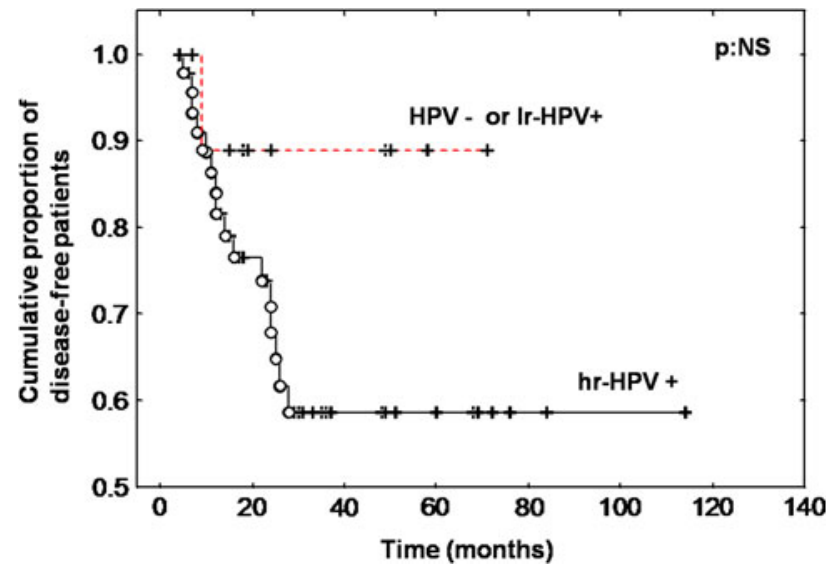

Fig. 2 Disease-free survival curves for high-risk HPV+ (hrHPV+) versus $\mathrm{HPV}-$ and low-risk HPV+ $(\mathrm{lrHPV}+)$ patients. The $P$-value is not significant (NS)

hrHPV+ tumour group $(79.5 \%$ were p21 + ) and in the $\mathrm{HPV}-$ or lrHPV+ tumour group $(82 \%$ were p21+) (Table 2).

p53 expression as determined by IHC

Due to insufficient biopsy material, two cases could not be analysed for p53 expression. Overexpression of p53 was detected in 27 of the $73(37 \%)$ hypopharyngeal SCC
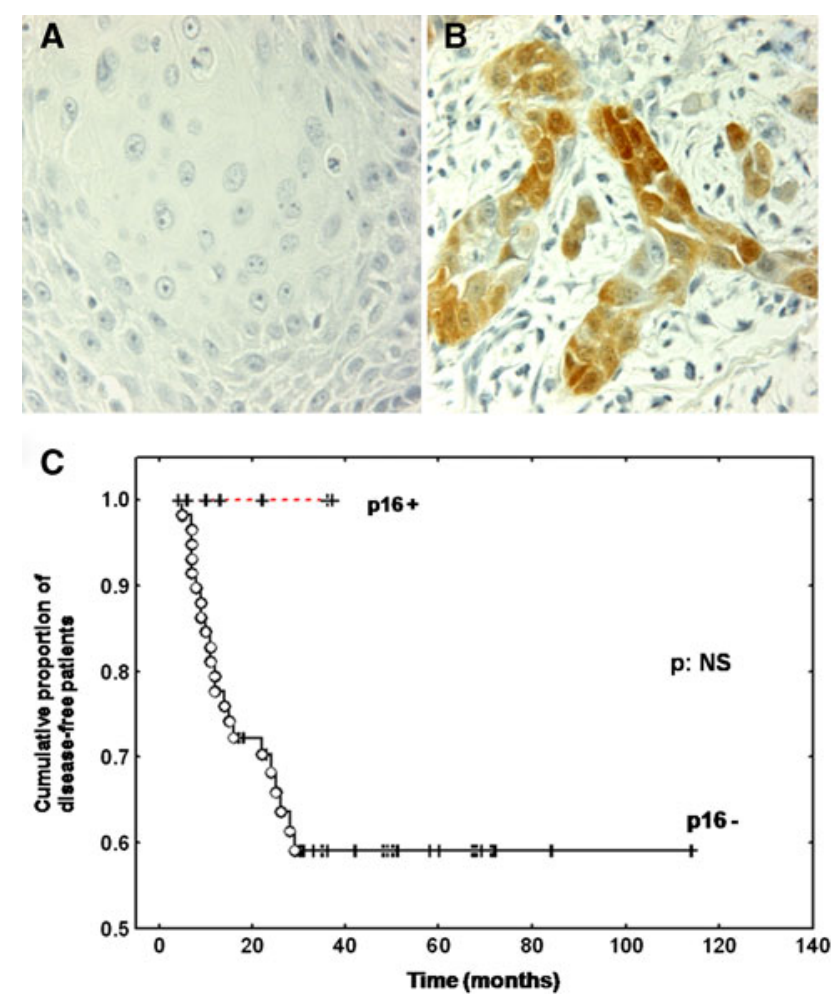

Fig. 3 Typical p16 immunohistochemical staining profile for stage IV hypopharyngeal carcinomas (a: p16 non-expressor and b: p16 expressor). c shows the survival curves for p16-expressor versus p16-nonexpressor patients. The $P$-value is not significant (NS). Magnification $\mathbf{a}-\mathbf{b} \times 320$

samples that were analysed (Fig. 5). In the HPV- group, $36 \%$ of tumours (4/11) were found to overexpress p53, whereas $33 \%$ of tumours in the hrHPV+ group (16/48 cases) were found to overexpress p53 (Fisher's exact test, NS) (Table 2). As shown in Fig. 5b, p53 expression was assessed by the presence of typical nuclear staining. Survival analysis showed that p53 overexpression was significantly associated with worse prognosis in our homogenous series of stage IV hypopharyngeal SCC patients (Fig. 5c). The 5-year disease-free survival was $73 \%$ in p53- tumours versus $48 \%$ in p53+ tumours (log-rank test, $P=0.008$ ).

We carried out multivariate Cox regression models on clinical and biological variables (age, node status, capsular effraction, QPCR-PCR HPV, p16, p21, p53 expression). These analyses confirmed univariate results, i.e. only p53 brought significant contribution to prognosis. No other clinical or biological variable was able to significantly contribute to the model (data not shown).

\section{Discussion}

Epidemiological, molecular and clinical evidence indicate that hrHPV may play a pivotal role in the development of HNSCCs. However, most previous studies on this topic 

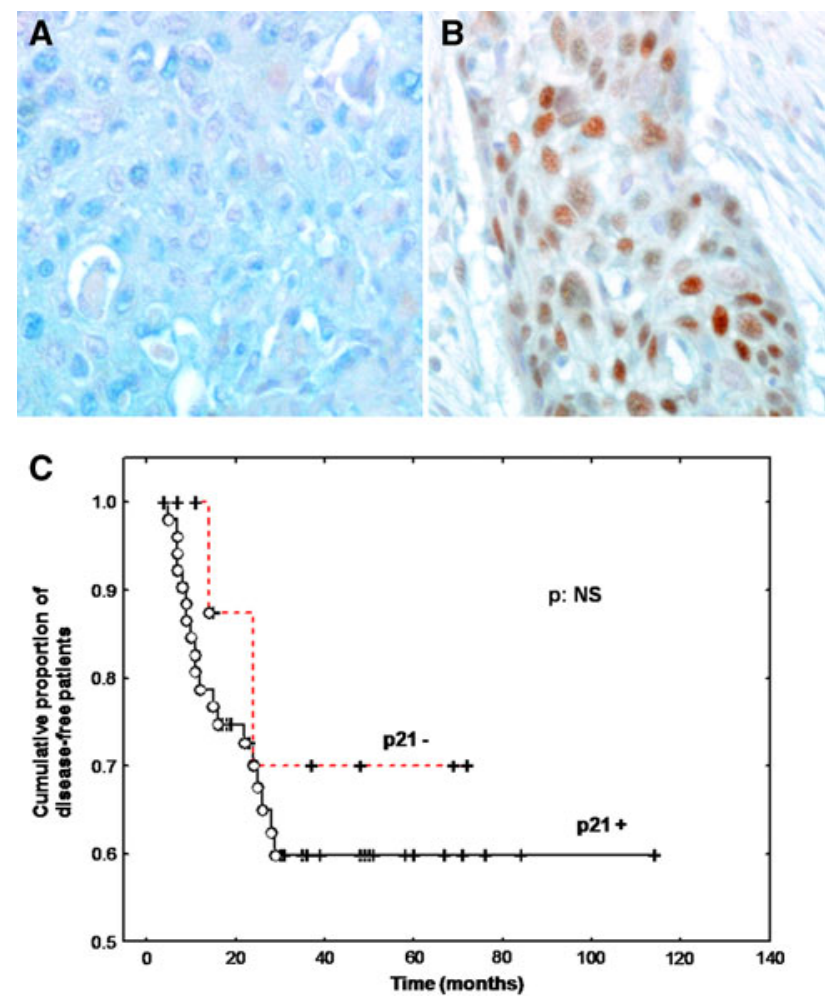

Fig. 4 Typical p21 immunohistochemical staining profile for stage IV hypopharyngeal carcinomas (a: p21 non-expressor and b: p21 expressor). c shows the survival curves for $\mathrm{p} 21$-expressor versus p16-nonexpressor patients. The $P$-value is not significant (NS). Magnification $\mathbf{a}-\mathbf{b} \times 320$

have focused on OSCCs (Dahlstrand et al. 2008). D'Souza et al. (2007) showed that oral HPV infection was significantly associated with OSCC among patients with or without a history of tobacco and alcohol use. In a Swedish study, oral hrHPV infection was associated with dramatically increased risk of OSCC development (odds ratio 230) after adjusting for alcohol and tobacco consumption (Hansson et al. 2005). Several studies have suggested that oral HPV infection is sexually acquired (D'Souza et al. 2007; Hansson et al. 2005). However, even though oralgenital contact may be implicated in oral HPV transmission, transmission through direct mouth to mouth contact or other means could not be excluded (Saussez et al. 2008). Tonsillar crypts, which have an oropharyngeal sublocation that is histologically similar to the transformation zone of the uterine cervix, seem to be particularly susceptible to HPV-induced malignant transformation (D'Souza et al. 2007; Hansson et al. 2005; Psyrri and DiMaio 2008). HPVassociated carcinomas tend to be poorly differentiated, and HPV-16 is the most prevalent genotype that is present in this type of tumour. Moreover, other studies have demonstrated that patients with hrHPV+ OSCC have a better prognosis than hrHPV- OSCC patients and that specific subgroups of hrHPVs+ tumours (specifically, those that have certain molecular profiles, such as p16 overexpression
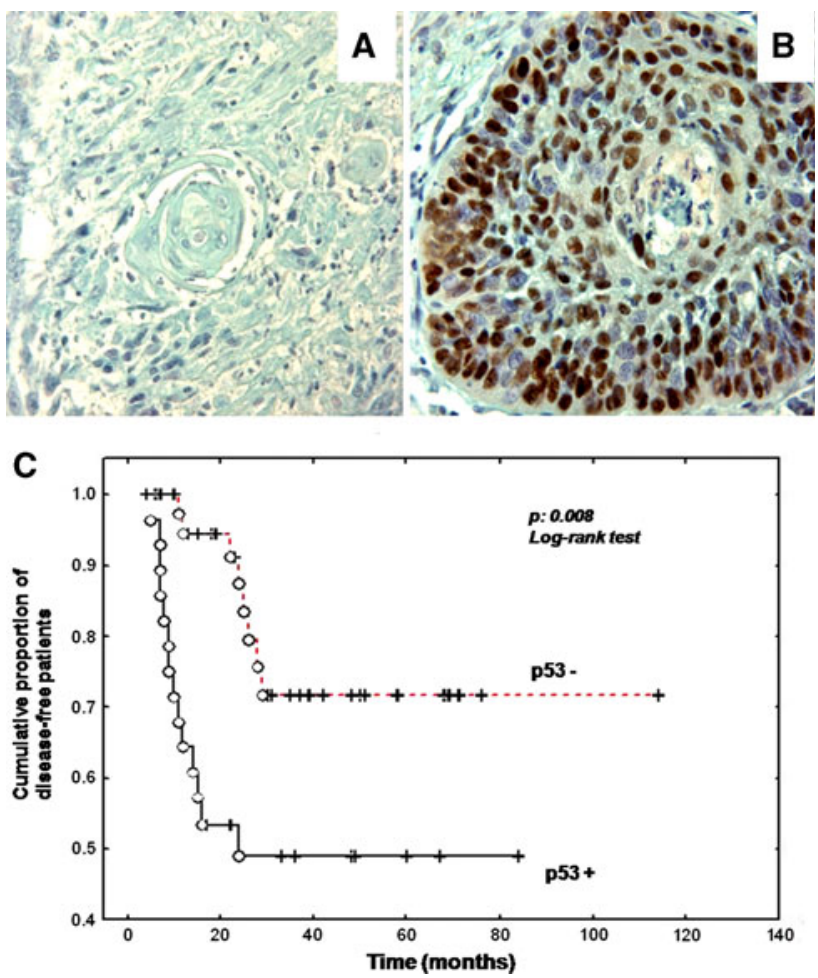

Fig. 5 Typical p53 immunohistochemical staining profile for stage IV hypopharyngeal carcinomas (a: p53 non-expressor and b: p53 expressor). c shows the survival curves of $\mathrm{p} 53$-expressor versus p53-non-expressor patients. Magnification $\mathbf{a}-\mathbf{b} \times 320$

or p53 underexpression) were associated with better disease-free survival rates (Reimers et al. 2007; Ritchie et al. 2003; Smith et al. 2008; Weinberger et al. 2006). Several studies have also demonstrated the involvement of HPV infections in laryngeal and sino-nasal tumours (Aaltonen et al. 2002; Alos et al. 2009). Very few publications have focused on HPV positivity in hypopharyngeal cancers. Our results revealed that a large percentage $(82 \%)$ of the tumour specimens that we studied were hrHPV+. These findings are in accordance with a previous study by Morgan et al. (1991) who described for the first time that $75 \%$ of the 16 pharyngolaryngeal carcinomas in their series were HPV+. Another study found a lower HPV positivity rate (46\%) in the tumours of 78 previously untreated patients with laryngeal and hypopharyngeal carcinomas (Clayman et al. 1994). The authors concluded that HPV+ tumours represented a biologically distinct subset of tumours that had a worse prognosis than HPV- tumours (Clayman et al. 1994). In contrast to our results, Paz et al. (1997) did not identify any HPV+ tumours in a small series of 7 hypopharyngeal carcinoma patients. This finding might be explained by the fact that, in our study, we used a very sensitive (10-100 copies per PCR reaction) and type-specific real-time quantitative PCR analysis with a short amplification product $(60-80 \mathrm{bp})$, which is less vulnerable to the 
presence of degraded DNA in paraffin-embedded specimens than some other analytical methods. We demonstrated for the first time in a large series of stage IV HSCC patients that $82 \%$ of tumours had hrHPV DNA present. Furthermore, a significant number of patients (20\%) were infected with multiple types of hrHPV.

The significance of hrHPV infection and its relationship with patient prognosis is still an important matter of debate, especially considering the contradictory results that are present in different studies in the literature (Dahlstrand et al. 2008; Rosenquist et al. 2007). Several studies have demonstrated that the presence of HPV DNA in tonsillar carcinomas is a favourable prognostic factor with regard to clinical outcomes (recurrence and survival) (Dahlstrand et al. 2008). In contrast, a recent study by Rosenquist et al. (2007) showed that among patients with oral and oropharyngeal tumours, the hrHPV+ subgroup had a higher risk of recurrence or development of second primary tumour, but had a lower risk of death due to intercurrent disease, when compared to the hrHPV - group. In the current study, we were not able to find a significant correlation between hrHPV positivity and clinical data (specifically, tumour differentiation, lymph node involvement, capsular effraction or laryngeal cartilage invasion). However, our hrHPV+ group had a higher recurrence rate $(32 \%, 16 / 50$ cases) than the HPV - and lrHPV+ groups (8\%, 1/11 cases). This finding suggests that hrHPV positivity may be associated with an elevated risk of tumour recurrence.

The oncoprotein E6 targets p53 for ubiquitin-mediated degradation, while E7 binds to and inactivates the protein product of the tumour-suppressor gene $\mathrm{Rb}$ (Munger et al. 1989; Scheffner et al. 1990). The p16 tumour suppressor gene is often upregulated in HPV-induced cancers because it is negatively regulated by pRb (Hara et al. 1996). In contrast, loss of p16 protein expression is a common and early event in the development of tobacco-related HNSCCs. Thus, tobacco/alcohol-associated HNSCCs appear to be associated with p16 downregulation and the TP53 gene mutation that results in the accumulation of p53 (corresponding to 553 overexpression) (Psyrri and DiMaio 2008). Our results demonstrate that the biology of hypopharyngeal SCCs is probably more complex than previously thought and that their tumourigenesis could involve several different molecular pathways. Our results illustrate the typical tobacco-related loss of p16 expression; only $11 \%$ of the tumours in our case series, which were obtained from patients who used tobacco and alcohol, expressed this protein. Thus, p16 overexpression seems to be associated with an improved prognosis. We did not find a statistical difference between the survival curves of p16+ expressors and non-expressors (seven p16-expressor patients did not suffer recurrence). The results of our study show that hrHPV tumours have higher recurrence rates if these tumours also overexpress $\mathrm{p} 53$ (when compared to p53-/HPV - tumours) (Table 2). Therefore, our findings suggest that p53 status plays a more significant role in predicting recurrence than hrHPV status. This finding was also recently described by Smith et al. (2008) in a large series of 294 HNSCC patients.

The strengths of our study include that we had a relatively large, very homogenous case series comprised of patients with stage IV hypopharyngeal carcinoma with similar tobacco and alcohol habits. We were also able to study the both independent and combined effects of p16, p21, p53 and HPV status with regard to clinical outcomes (recurrence and survival). Another strength of this study was that we had very good follow-up; we had recurrence and survival data with a significant follow-up period available for $96 \%$ of our patients. Finally, we emphasise that our hypopharyngeal carcinoma series is composed of only resectable stage IV (60/75 cases presented negative surgical margins) hypopharyngeal carcinomas. This finding likely explains the relatively good overall survival rates for stage IV hypopharyngeal carcinoma that we observed among this patient group.

Conflict of interest statement All authors disclose no financial and personal relationships with other people or organisations that could inapropriately influence (bias) the work.

Open Access This article is distributed under the terms of the Creative Commons Attribution Noncommercial License which permits any noncommercial use, distribution, and reproduction in any medium, provided the original author(s) and source are credited.

\section{References}

Aaltonen L-M, Rihkanen H, Vaheri A (2002) Human papillomavirus in larynx. The Laryngoscope 112:700-707

Alos L, Moyona S, Nadal A et al (2009) Human papillomaviruses are identified in a subgroup of sinonasal squamous cell carcinomas with favorable outcome. Cancer 115:2701-2709

Arbyn M, Benoy I, Simoens C et al (2009) Prevaccination distribution of human papillomavirus types in women attending at cervical cancer screening in Belgium. Cancer Epidemiol Biomarkers Prev $18: 321-330$

Begum S, Gillison ML, Ansari-Lari MA et al (2003) Detection for papillomavirus in cervical lymph nodes: a highly effective strategy for localizing site of tumor origin. Clin Cancer Res 9:6469-6475

Bernier J, Domenge C, Ozsahin M et al (2004) Postoperative irradiation with or without concomitant chemotherapy for locally advanced head and neck cancer. N Engl J Med 350:1945-1952

Clayman GL, Stewart MG, Weber RS et al (1994) Human papillomavirus in laryngeal and hypopharyngeal carcinomas. Relationship to survival. Arch Otolaryngol Head and Neck Surg 120:743-748

Cooper JS, Pajak TF, Forastiere AA et al (2004) Postoperative concurrent radiotherapy and chemotherapy for high-risk squamous-cell carcinoma of the head and neck. N Engl J Med 350:1937-1944

D'Souza G, Kreimer AR, Viscidi R et al (2007) Case-control study of human papillomavirus and oropharyngeal cancer. N Engl J Med 356:1944-1956 
Dahlstrand H, Näsman A, Romanitan M et al (2008) Human papillomavirus accounts both for increased incidence and better prognosis in tonsillar cancer. Anticancer Res 28:1133-1138

Depuydt CE, Benoy IH, Bailleul EJ et al (2006) Improved endocervical sampling and HPV viral load detection by Cervex-Brush Combi. Cytopathology 17:374-381

Depuydt CE, Boulet GA, Horvath CA et al (2007) Comparison of MY09/11 consensus PCR and type-specific PCRs in the detection of oncogenic HPV types. J Cell Mol Med 11:881-891

Grandis JR, Pietenpol JA, Greeberger JS et al (2004) Head and neck cancer: meeting summary and research opportunities. Cancer Res 64:126-129

Hansson BG, Rosenquist K, Antonsson A et al (2005) Strong association between infection with human papillomavirus and oral and oropharyngeal squamous cell carcinoma: a population-based case-control study in southern Sweden. Acta Otolaryngol 125:1337-1344

Hara E, Smith R, Parry D et al (1996) Regulation of p16CDKN2 expression and its implications for cell immortalization and senescence. Mol Cell Biol 16:859-867

Hunter KD, Parkinson EK, Harrison PR (2005) Profiling early head and neck cancer. Nat Rev Cancer 5:127-135

Hyams VJ, Batsakis JG, Michaels L (eds) (1988) Tumors of the upper respiratory tract and ear. Atlas of tumor pathology. Armed Forces Institute of Pathology, Washington, DC

Kumar B, Cordell KG, Lee JS et al (2007) Response to therapy and outcomes in oropharyngeal cancer are associated with biomarkers including human papillomavirus, epidermal growth factor receptor, gender and smoking. Int J Radiat Oncol Biol Phys 69:109-111

Morgan DW, Abdullah V, Quiney R et al (1991) Human papilloma virus and carcinoma of the laryngopharynx. J Laryngol Otol 105:288-290

Munger K, Werness BA, Dyson N et al (1989) Complex formation of human papillomavirus E7 proteins with the retinoblastoma tumor suppressor gene product. EMBO J 8:4099-4105

Paz IB, Cook N, Odom-Maryon T et al (1997) Human papillomavirus (HPV) in head and neck cancer. An association of HPV 16 with squamous cell carcinoma of Waldeyer's tonsillar ring. Cancer 79:595-604
Psyrri A, DiMaio D (2008) Human papillomavirus and head and neck cancer. Nat Clin Pract Oncol 5:24-31

Reed AL, Califano J, Cairns P et al (1996) High frequency of p16 (CDKN2/MTS-1/INK4A) inactivation in head and neck squamous cell carcinoma. Cancer Res 56:3630-3633

Reimers N, Kasper HU, Weissenborn SJ et al (2007) Combined analysis of HPV-DNA, p16 and EGFR expression to predict prognosis in oropharyngeal cancer. Int J Cancer 120:1731-1738

Ritchie JM, Smith EM, Summersgill KF et al (2003) Human papillomavirus infection as a prognostic factor in carcinomas of the oral cavity and oropharynx. Int J Cancer 104:336-344

Rosenquist K, Wennerberg J, Annertz K et al (2007) Recurrence in patients with oral and oropharyngeal squamous cell carcinoma: human papillomavirus and other risk factors. Acta Otolaryngol 127:980-987

Saussez S, Decaestecker C, Lorfevre F et al (2008) Increased expression and altered intracellular distribution of adhesion/growthregulatory lectins galectins- 1 and -7 during tumour progression in hypopharyngeal and laryngeal squamous cell carcinomas. Histopathology 52:483-493

Schantz SP, Harrison LB, Forastiere AA (1997) Tumors of the nasal cavity and paranasal sinuses, nasopharynx, oral cavity, and oropharynx. In: De Vita VT Jr, Hellman S, Rosenberg SA (eds) Cancer: principles and practice of oncology, vol 1, 5th edn. JP Lippincott, Philadelphia, pp 741-847

Scheffner M, Werness BA, Huibregtse JM et al (1990) The E6 oncoprotein encoded by human papillomavirus types 16 and 18 promotes the degradation of p53. Cell 63:1129-1136

Smith EM, Wang D, Rubenstein LM et al (2008) Association between p53 and human papillomavirus in head and neck cancer survival. Cancer Epidemiol Biomarkers Prev 17:421-427

Weinberger PM, Yu Z, Haffty BG et al (2006) Molecular classification identifies a subset of human papillomavirus-associated oropharyngeal cancers with favorable prognosis. J Clin Oncol 24:736747

Wittekind C, Greene FL, Hutter RRP et al. (2004) TNM Atlas, 6th Edn. UICC, Berlin 